\title{
Sonic hedgehog signaling proteins and ATP-binding cassette G2 are aberrantly expressed in diffuse large B-Cell lymphoma
}

\author{
Ji Eun Kim ${ }^{1, *}$, Rajesh R Singh ${ }^{1}$, Jeong Hee Cho-Vega ${ }^{2}$, Elias Drakos ${ }^{1}$, Yogesh Davuluri ${ }^{1}$, \\ Faisal A Khokhar ${ }^{1}$, Luis Fayad ${ }^{3}$, L Jeffrey Medeiros ${ }^{1}$ and Francisco Vega ${ }^{1}$ \\ ${ }^{1}$ Department of Hematopathology, The University of Texas MD Anderson Cancer Center, Houston, TX, USA; \\ ${ }^{2}$ Department of Pathology, The University of Texas MD Anderson Cancer Center, Houston, TX, USA and \\ ${ }^{3}$ Department of Lymphoma/Myeloma, The University of Texas MD Anderson Cancer Center, Houston, TX, \\ $U S A$
}

Dysregulation of the sonic hedgehog (SHH) signaling pathway has been shown in several cancer types, but has not been explored in diffuse large B-cell lymphoma. We assessed 67 cases of diffuse large B-cell lymphoma for expression of SHH (ligand), GLI1, GLI2 and GLI3 (transcriptional effectors of SHH signaling), and the ATPbinding cassette (ABC)G2 (a downstream target of SHH signaling), using immunohistochemistry. For comparison, we assessed the expression levels of these proteins in 28 cases of follicular lymphoma, 5 chronic lymphocytic leukemia/small lymphocytic lymphoma, and 5 reactive lymph nodes. In diffuse large B-cell lymphoma, SHH was expressed in 61 of $67(91 \%)$ cases, GLI1 in 62 of $67(93 \%)$, GLI2 in 41 of 56 (73\%), and GLI3 in 22 of $56(39 \%)$. Expression of ABCG2 was detected in 52 of $55(95 \%)$ cases and was high in $15(27 \%)$ cases. SHH expression positively correlated with expression levels of ABCG2 $(P=0.05)$. Patients with diffuse large $B-$ cell lymphoma with high ABCG2 expression showed significantly shorter overall survival $(P=0.031)$ and failurefree survival $(P=0.029)$ compared with patients with tumors with low or no expression of ABCG2. Diffuse large B-cell lymphomas expressed SHH, and GLI1, GLI2, and GLI3 more frequently and more intensely than cases of follicular lymphoma or chronic lymphocytic leukemia/small lymphocytic lymphoma. In conclusion, our data show that SHH signaling proteins and ABCG2 are aberrantly expressed in diffuse large B-cell lymphoma and that ABCG2 expression has prognostic implications. These findings also provide evidence that dysregulation of the SHH pathway may be involved in the pathogenesis of diffuse large B-cell lymphoma.

Modern Pathology (2009) 22, 1312-1320; doi:10.1038/modpathol.2009.98; published online 10 July 2009

Keywords: sonic hedgehog (SHH) signaling pathway; GLI; diffuse large B-cell lymphoma; ABCG2

Diffuse large B-cell lymphoma is one of the most common lymphoma types in adults. ${ }^{1}$ The pathogenesis of diffuse large B-cell lymphoma is both complex and heterogeneous, and many pathogenetic mechanisms remain largely unknown. Although

Correspondence: Dr F Vega, MD, PhD, Department of Hematopathology, Unit 72, The University of Texas MD Anderson Cancer Center, Box 72, 1515 Holcombe BLVD, Houston, TX 77030, USA. E-mail: fvegava@mdanderson.org

This paper was presented in part at the United States and Canadian Academy of Pathology (USCAP) Annual meeting, Boston, MA, 7-13 March 2009.

${ }^{*}$ Dr JE Kim did this work as a visiting scientist supported by the Department of Pathology, Seoul National University, Boramae Hospital, Seoul, Korea.

Received 28 March 2009; revised 28 May 2009; accepted 29 May 2009; published online 10 July 2009 many diffuse large B-cell lymphoma patients respond to conventional anthracyclin-based chemotherapy, approximately half of all patients eventually die of their disease. ${ }^{1}$ New advances in understanding the molecular pathogenesis of diffuse large B-cell lymphoma are needed and will likely have significant implications for developing new therapeutic approaches.

The Hedgehog protein family is a group of secreted signaling molecules that is critical for normal mammalian development. ${ }^{2}$ Recent studies also implicate these proteins in cell fate determination and self-renewal of the hematopoietic system, and sonic hedgehog ( $\mathrm{SHH}$ ) is one of the survival signals provided by follicular dendritic cells to prevent apoptosis in germinal center B cells.,4 SHH ligand interacts with a receptor complex 
composed of two proteins, patched (PTCH) and smoothened (SMO). PTCH is the SHH ligand-binding subunit and SMO is the signal transduction component. In the absence of SHH, PTCH inhibits SMO. Once SHH binds PTCH, this inhibition is released allowing SMO to transduce the SHH signal, mediated by the glioma-associated oncogene homolog (GLI) transcription factors (GLI1, 2, and 3). ${ }^{5}$ Although both GLI2 and GLI3 have transcriptional activation and repression properties, GLI1 is a strong positive regulator of $\mathrm{SHH}$ transcriptional targets and is itself a transcriptional target of $\mathrm{SHH}^{6-9}$

Chemotherapy resistance remains a significant impediment to the successful treatment of patients with diffuse large B-cell lymphoma. The multidrug resistance phenotype is often associated with increased expression of ATP-binding cassette (ABC) transporters that mediate energy-dependent transport of substrate drugs out of the cell against a concentration gradient. ${ }^{10}$ Drugs that are affected by classical multidrug resistance include the vinca alkaloids (vincristine and vinblastine), the anthracyclines (doxorubicin and daunorubicin), the RNA transcription inhibitor, actinomycin-D, and the microtubule-stabilizing drug paclitaxel. ${ }^{11}$ One of the major multidrug resistant pumps is ABCG2, also known as breast cancer resistance protein /placental ABC protein/ mitoxantrone resistance protein. Recent studies suggest that $\mathrm{SHH}$ signaling activation upregulates the expression of ABCG2 in several cancers. ${ }^{12,13}$

Although inappropriate activation of the $\mathrm{SHH}$ signaling pathway has been shown in many cancers, $\mathrm{SHH}$ proteins have not been assessed in diffuse large B-cell lymphoma, nor have ABCG2 protein levels been assessed in these tumors. Here, we investigated expression of SHH signaling proteins and ABCG2 in diffuse large B-cell lymphoma. We compared these results with those obtained in cases of follicular lymphoma and chronic lymphocytic leukemia/small lymphocytic lymphoma, two common types of B-cell lymphoma, and in reactive lymph nodes. Our findings show that $\mathrm{SHH}$ signaling proteins are aberrantly expressed in diffuse large B-cell lymphoma. We also found that high ABCG2 expression levels correlate with shorter overall and failure-free survival in diffuse large B-cell lymphoma patients. In aggregate, the findings suggest that the $\mathrm{SHH}$ pathway is dysregulated in diffuse large B-cell lymphoma.

\section{Materials and methods}

\section{Patient Samples}

The study group included 67 diffuse large B-cell lymphomas, 28 follicular lymphomas, and 5 cases of chronic lymphocytic leukemia/small lymphocytic lymphoma accessioned at The University of Texas MD Anderson Cancer Center between 1987 and 2006. The diagnosis and grading of these tumors was based on criteria according to the World Health Organization classification. According to the expres- sion of CD10, BCL-6, and MUM-1, cases were classified as either germinal center or non-germinal center type using the system of Hans et al. ${ }^{14}$ Clinical data, such as age, Ann Arbor stage, international prognostic index, and survival time were reviewed and their association with protein expression levels was analyzed statistically. The 67 diffuse large B-cell lymphoma patients in this study included 37 men and 30 women, ages 29-92 years (median, 63 years). At time of diagnosis, bone marrow involvement was present in 46 . A total of $40(60 \%)$ cases were classified as germinal center type and $27(40 \%)$ as non-germinal center type. Diffuse large B-cell lymphomas with substantial necrosis, a starry sky pattern or numerous apoptotic bodies, or a high proliferation rate $(>70 \%)$ as detected by MIB1 (Ki-67), were considered as tumors with aggressive or high-grade features. A total of $39(61 \%)$ diffuse large B-cell lymphomas in this study had high-grade features. The study group also included 5 cases of T cell/histiocyte-rich large B-cell lymphoma. A total of $27(47 \%)$ patients had an International Prognostic Index $\geq 3$, and $27(47 \%)$ patients had an elevated serum LDH level. The median follow-up period was 35 months (range, 4-256 months). At the time of writing, 36 (58\%) patients were alive and $26(42 \%)$ patients died of disease. To study the baseline expression of $\mathrm{SHH}$ signaling proteins and ABCG2 in normal lymphoid cells, five reactive lymph nodes were also included.

\section{Cell Lines}

The following diffuse large B-cell lymphoma cell lines were used: Pfeiffer (ATCC, Manassas, VA), DOHH2 (DSMZ, Braunschweig, Germany), MS and MCA (both kindly provided by Dr Richard Ford, UT MD Anderson Cancer Center). ${ }^{15}$

\section{Immunohistochemistry}

Immunohistochemical analysis was performed as previously described, ${ }^{16}$ using the antibodies and staining conditions listed in Table 1. Briefly, all

Table 1 Antibodies used for immunohistochemistry

\begin{tabular}{lllcl}
\hline Antibody & Clone & Manufacturer & Dilution & Incubation \\
\hline SHH & H-160 & Santa Cruz & $1: 150$ & RT, 1 h \\
GLI1 & H-300 & Santa Cruz & $1: 200$ & RT, 1h \\
GLI2 & NA & Abcam & $1: 200$ & RT, 1.5 h \\
GLI3 & H-280 & Santa Cruz & $1: 150$ & RT, 1 h \\
ABCG2 & BXP-21 & Santa Cruz & $1: 400$ & RT, 1 h \\
CD10 & 56C6 & Lab Vision & $1: 50$ & RT, 1 h \\
CD20 & L26 & Dako & $1: 1000$ & RT, 1 h \\
CD79a & JGB117 & Dako & $1: 50$ & RT, 1 h \\
BCL-6 & PG-B6P & Dako & $1: 200$ & RT, 1.5 h \\
MUM-1 & IRF-4 & Santa Cruz & $1: 400$ & RT, 1 h \\
PAX-5 & 24 & BD Biosciences & $1: 35$ & RT, 1 h \\
Ki-67 & MIB-1 & Dako & $1: 50$ & RT, 1.5 h \\
& & & &
\end{tabular}

NA, not available, RT, room temperature. 
tissue sections underwent heat-induced epitope retrieval in pH 6.0 citrate buffer (Dako, Carpinteria, CA). Endogenous peroxidase was blocked by $3 \%$ $\mathrm{H}_{2} \mathrm{O}_{2}$ solution for $10 \mathrm{~min}$. To avoid nonspecific binding of primary antibodies, serum-free blocking solution (Dako) was applied for $40 \mathrm{~min}$ at room temperature. Detection was performed using the LSAB plus-streptavidin-HRP system (Dako). Cases were considered positive if $10 \%$ or more (by estimate) of the tumor cells were stained with an antibody, as previously described. ${ }^{17}$ Protein expression was scored as negative, low, or high depending on the staining signal intensity. Expression of ABCG2 in the tumor cells was compared with that seen in endothelial cells; if higher, then expression of ABCG2 was considered high and if lower or equal, then expression of ABCG2 was considered low. Appropriate external controls were used for each antibody.

\section{Western Blot Analysis}

Western blotting was performed as described previously. ${ }^{16}$ Antibodies used were as follows: $\mathrm{SHH}$, SMO, GLI1, and PTCH (Santa Cruz Biotechnology, Santa Cruz, CA), and $\beta$-actin (Sigma, St Louis, MO). Reactions were visualized with suitable secondary antibodies conjugated with horseradish peroxidase using enhanced chemiluminescence reagents (Amersham Pharmacy, Piscataway, NJ).

\section{Double Immunofluorescence Labeling}

Double immunofluorescence labeling was performed using paraffin-embedded tissue sections of a reactive lymph node for GLI3 and CD21. The slides were incubated overnight at $4{ }^{\circ} \mathrm{C}$ with an anti-CD21 antibody (Dako), and for $2 \mathrm{~h}$ at room temperature with an anti-GLI3 antibody (Santa Cruz Biotechnology). Alexa Fluor 594 (red) goat antibody IgG conjugate and Alexa Fluor 488 (green) goat antibody anti-rabbit IgG conjugate antibody (Molecular probes, Eugene, OR) were used as secondary antibodies. The nuclei were counterstained with $4^{\prime}-6^{\prime}$ diamidino-2-phenylindole (DAPI). After aqueous mounting, the slides were observed using a fluorescence microscope (Olympus BX51, Tokyo, Japan).

\section{Statistical Analysis}

Statistical analysis was performed using the SPSS 16.0 software (Chicago, IL). $\chi^{2}$ and Fisher's exact tests were used to correlate protein expression with clinicopathological variables. The Kaplan-Meier method and the log-rank test were used to estimate and compare overall survival and failure-free survival. Statistical significance was defined as a $P$-value of $\leq 0.05$.

\section{Results}

SHH Signaling Proteins are Infrequently Expressed or Expressed at Low Levels in Germinal Center Cells

We investigated the expression levels of SHH, GLI1, 2 and 3, and ABCG2 in normal B cells of five reactive lymph node biopsy specimens. The lymph node cell types expressing these proteins are summarized in Table 2 (Figure 1).

Sonic hedgehog was positive in the cytoplasm of follicular dendritic cells, endothelial cells, and macrophages, but not in germinal center lymphocytes (Figure 1a, inset). Germinal center lymphocytes, follicular dendritic cells, and endothelial cells were negative for GLI1 (Figure 1b). Weak nuclear expression of GLI2 was detected in paracortical T cells, and in some centroblasts and centrocytes surrounding follicular dendritic cells within germinal centers (Figure 1c). Expression of GLI3 with characteristic positivity restricted to the nuclear membrane was detected in follicular dendritic cells and endothelial cells, but not in germinal center lymphocytes (Figure 1d). The expression of GLI3 by follicular dendritic cells was confirmed by double immunofluorescence labeling using antibodies against CD21 and GLI3 (Figure 1e). These findings indicate that GLI2 is the predominant $\mathrm{SHH}$ transcription factor expressed at low levels in germinal center lymphocytes. Low expression of ABCG2 was detected in a subset of germinal center lymphocytes, endothelial cells, and some paracortical immunoblasts (Figure 1f).

SHH Signaling Proteins are Infrequently Expressed or Expressed at Low Levels in Follicular Lymphoma and Chronic Lymphocytic Leukemia/Small Lymphocytic Lymphoma

A summary of the immunohistochemical findings in follicular lymphoma is presented in Table 2 (Figures 2a-e). We detected low expression of $\mathrm{SHH}$ and GLI1 proteins in a subset of centroblasts in 8 (29\%) and 2 (7\%) of 28 follicular lymphoma tumors, respectively (Figures 2a and b). Low expression of GLI2 was found in five (18\%) and that of GLI3 in two $(7 \%)$ tumors (Figures 2c and $\mathrm{d}$ ). These results indicate that $\mathrm{SHH}$ signaling is expressed infrequently and at low levels in follicular lymphoma. Expression of cytoplasmic ABCG2 was detected in all the cases (Figure 2e), but high cytoplasmic positivity was seen in only two $(7 \%)$ tumors. No correlation between levels of expression of $\mathrm{SHH}$ signaling proteins and/or ABCG2 and histological grade, or with any of the other clinicopathological variables was noted.

To further assess the expression of $\mathrm{SHH}$ signaling proteins in low-grade lymphomas, we included in this study five lymph nodes involved by chronic lymphocytic leukemia/small lymphocytic lymphoma. Most of the chronic lymphocytic leukemia cells 
Table 2 Summary of the immunohistochemistry results

\begin{tabular}{|c|c|c|c|c|}
\hline & $\begin{array}{l}\text { Diffuse large B-cell } \\
\text { lymphoma }(\mathrm{n}=67)\end{array}$ & $\begin{array}{c}\text { Follicular } \\
\text { lymphoma }(\mathrm{n}=28)\end{array}$ & $\begin{array}{c}\text { Chronic lymphocytic } \\
\text { leukemia/small } \\
\text { lymphocytic lymphoma } \\
(\mathrm{n}=5)\end{array}$ & $\begin{array}{l}\text { Reactive lymph nodes } \\
\qquad(\mathrm{n}=5)\end{array}$ \\
\hline \multicolumn{5}{|l|}{$\mathrm{SHH}$} \\
\hline \multirow[t]{2}{*}{ Positivity } & 61/67 (91\%) & $8 / 28(29 \%)$ & $0 / 5^{\mathrm{a}}$ & \\
\hline & High: 33/67 (49\%) & High: $0 / 28$ & & \\
\hline Specificity & Tumor cells & Tumor cells & & $\begin{array}{l}\text { Follicular dendritic cells, } \\
\text { endothelial cells, macrophages }\end{array}$ \\
\hline \multicolumn{5}{|l|}{ GLI1 } \\
\hline \multirow[t]{2}{*}{ Positivity } & 62/67 (93\%) & $2 / 28(7 \%)$ & $0 / 5^{\mathrm{a}}$ & \\
\hline & High: 28/67(42\%) & High: 0/28 & & \\
\hline Specificity & Tumor cells & Tumor cells & & No positive cells \\
\hline \multicolumn{5}{|l|}{ GLI2 } \\
\hline \multirow[t]{2}{*}{ Positivity } & $41 / 56(73 \%)$ & $5 / 28(18 \%)$ & $0 / 5$ & \\
\hline & High: $15 / 56(27 \%)$ & High: $0 / 28$ & & \\
\hline Specificity & Tumor cells & Tumor cells & & $\begin{array}{l}\text { T cells, few germinal center cells } \\
\text { close to follicular dendritic cells }\end{array}$ \\
\hline \multicolumn{5}{|l|}{ GLI3 } \\
\hline \multirow[t]{2}{*}{ Positivity } & $22 / 56(39 \%)$ & $2 / 28(7 \%)$ & $0 / 5$ & \\
\hline & High: $2 / 56$ (4\%) & High: $0 / 28$ & & \\
\hline Specificity & Tumor cells & Tumor cells & & $\begin{array}{l}\text { Follicular dendritic cells and } \\
\text { endothelial cells (high) }\end{array}$ \\
\hline \multicolumn{5}{|l|}{ ABCG2 } \\
\hline \multirow[t]{2}{*}{ Positivity } & $52 / 55(95 \%)$ & $28 / 28(100 \%)$ & $0 / 5^{\mathrm{a}}$ & \\
\hline & High: 15/55 (27\%) & High: 2/28 (7\%) & & \\
\hline Specificity & Tumor cells & Tumor cells & & $\begin{array}{l}\text { Germinal center B cells, } \\
\text { endothelial cells (low) }\end{array}$ \\
\hline
\end{tabular}

${ }^{\mathrm{a}}$ Occasional prolymphocytes and paraimmunoblasts inside the proliferation centers were found to express low levels of SHH, GLI1, and ABCG2. In addition, occasional dendritic cells inside the proliferation centers and occasional paracortical stromal spindle cells were positive for SHH.
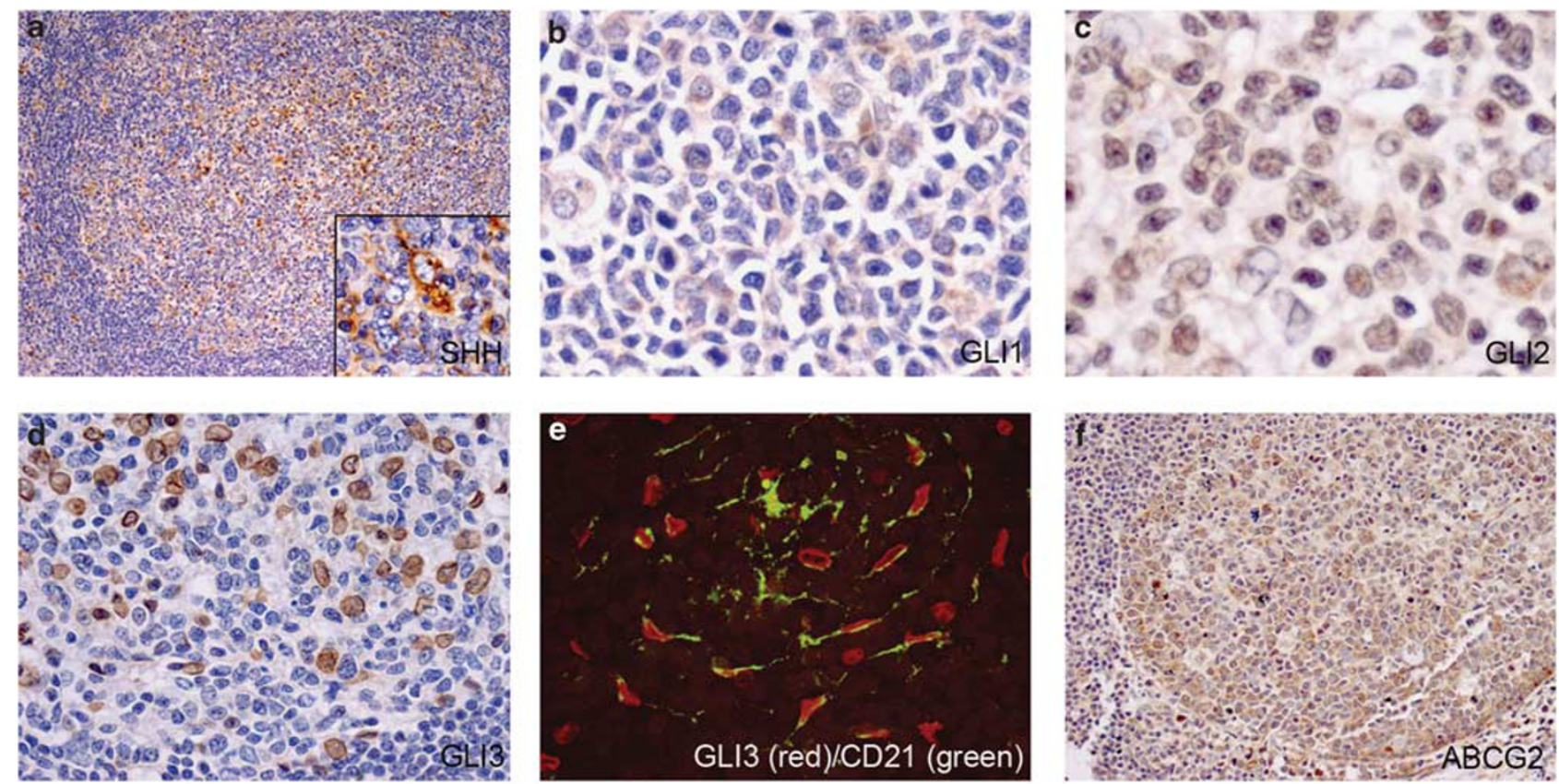

Figure 1 Sonic hedgehog signaling proteins and ABCG2 expression in germinal centers of reactive lymph nodes. (a) SHH is expressed in the cytoplasm of follicular dendritic cells (inset: high magnification). (b) No expression of GLI1 is detected in normal germinal center lymphocytes. (c) A subset of germinal center cells, in particular those surrounding follicular dendritic cells, express low levels of GLI2 with a nuclear pattern. (d) GLI3 (nuclear) is highly expressed by follicular dendritic cells and endothelial cells. (e) Double immunofluorescence analysis show that most of the cells expressing nuclear GLI3 (in red) also express CD21 (in green) and represent follicular dendritic cells. (f) Low cytoplasmic expression of ABCG2 is found in a subset of germinal center cells and endothelial cells. 

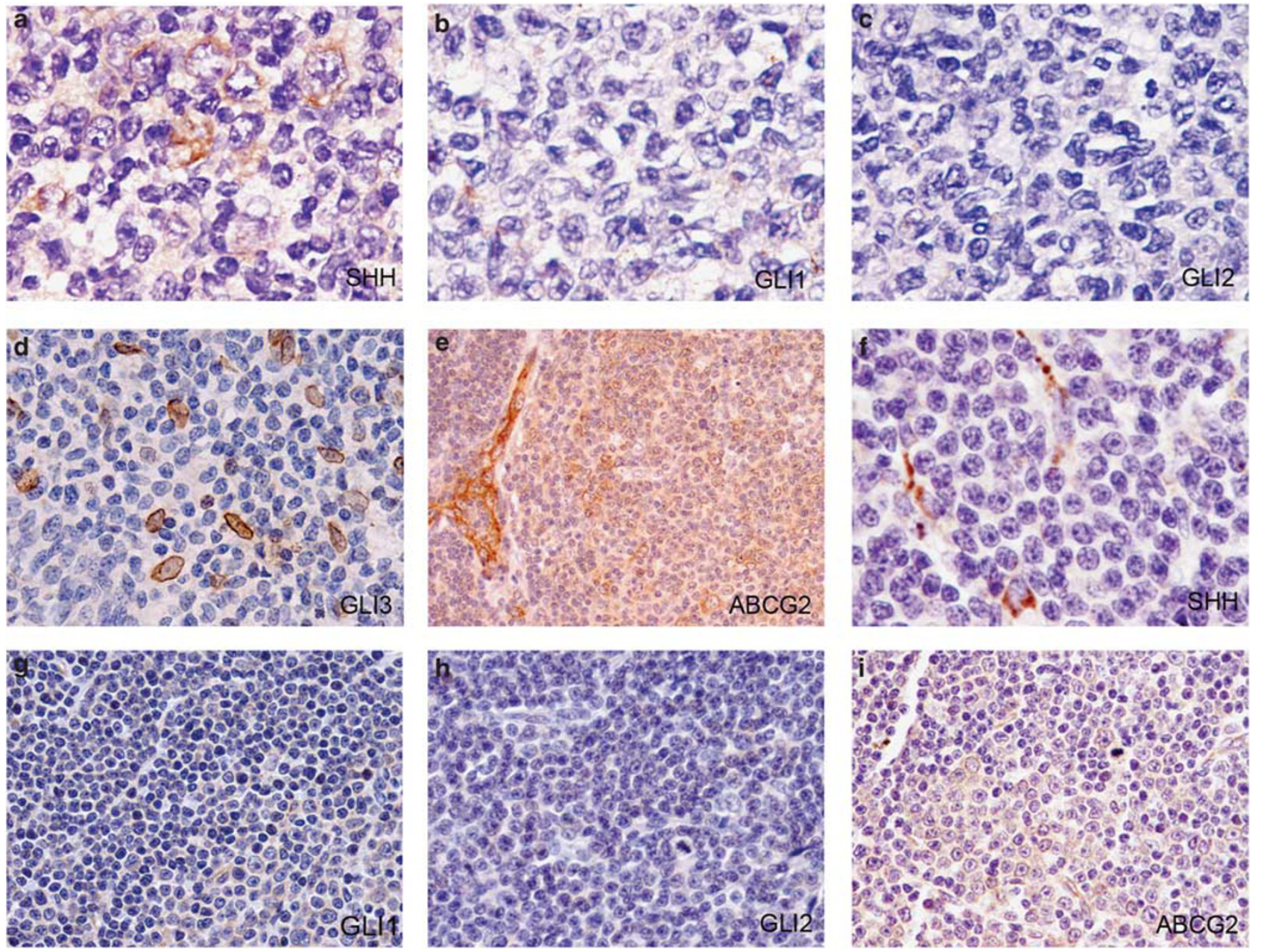

Figure 2 Sonic hedgehog signaling proteins and ABCG2 expression in follicular lymphoma and chronic lymphocytic leukemia/small lymphocytic lymphoma. (a) SHH is positive in a subset of follicular lymphomas, 8 of 28 cases in this study. Follicular dendritic cells are also positive (b, d). Rare follicular lymphoma cells are positive for GLI1 (b), GLI2 (c), and GLI3 (d). Follicular dendritic cells are also positive for GLI3 (d). Most cases of chronic lymphocytic leukemia/small lymphocytic lymphoma are negative for SHH (f), GLI1 (g), GLI2 (h), and GLI3 (not shown). Occasional paracortical stromal cells with spindle morphology are positive for SHH (f). (i) ABCG2 is expressed by a subset of prolymphocytes and paraimmunoblasts in chronic lymphocytic leukemia/small lymphocytic lymphoma at low levels.

were negative for SHH, GLI1, GLI2, GLI3, and ABCG2 (Figures 2f-i). Occasional prolymphocytes and paraimmunoblasts inside proliferation centers showed low levels of expression of SHH, GLI1, and ABCG2. In addition, occasional cells with dendritic cell morphology within the proliferation centers and occasional paracortical stromal spindle cells were positive for SHH, but not for GLI (Figure 2f).

\section{SHH Signaling Proteins are Frequently Highly Expressed in Diffuse Large B-Cell Lymphoma}

A summary of the immunohistochemistry results is presented in Table 2 (Figures 3a-e). SHH was expressed in the cytoplasm of 61 of 67 (91\%) diffuse large B-cell lymphomas and was high in $33(49 \%)$ cases (Figure 3a). GLI1 was expressed in nuclei in 62 of $67(93 \%)$ cases and was high in $28(42 \%)$ (Figure 3b). Nuclear GLI2 was detected in 41 of $56(73 \%)$ diffuse large B-cell lymphomas and was high in 15 $(27 \%)$, (Figure 3c) and GLI3 was expressed in nuclei in 22 of $56(39 \%)$ cases, and was high in $2(4 \%)$ (Figure 3d). ABCG2 was expressed in a cytoplasmic and membranous pattern in 52 of $55(95 \%)$ diffuse large B-cell lymphomas and was highly expressed in $15(27 \%)$ (Figure 3e).

Western blot analysis of diffuse large B-cell lymphoma cell lines showed high expression of SHH and SHH-related proteins confirming the immunohistochemical results (Figure 3f). These data indicate that diffuse large B-cell lymphoma cells produce $\mathrm{SHH}$ and provide support for the existence of a SHH signaling autocrine loop. As shown in Figure 3f, diffuse large B-cell lymphoma cell lines expressed both components of the $\mathrm{SHH}$ receptor, PTCH (ligand-binder subunit), and SMO (signal-transducer subunit), indicating that they can receive and transmit the $\mathrm{SHH}$ signal. 

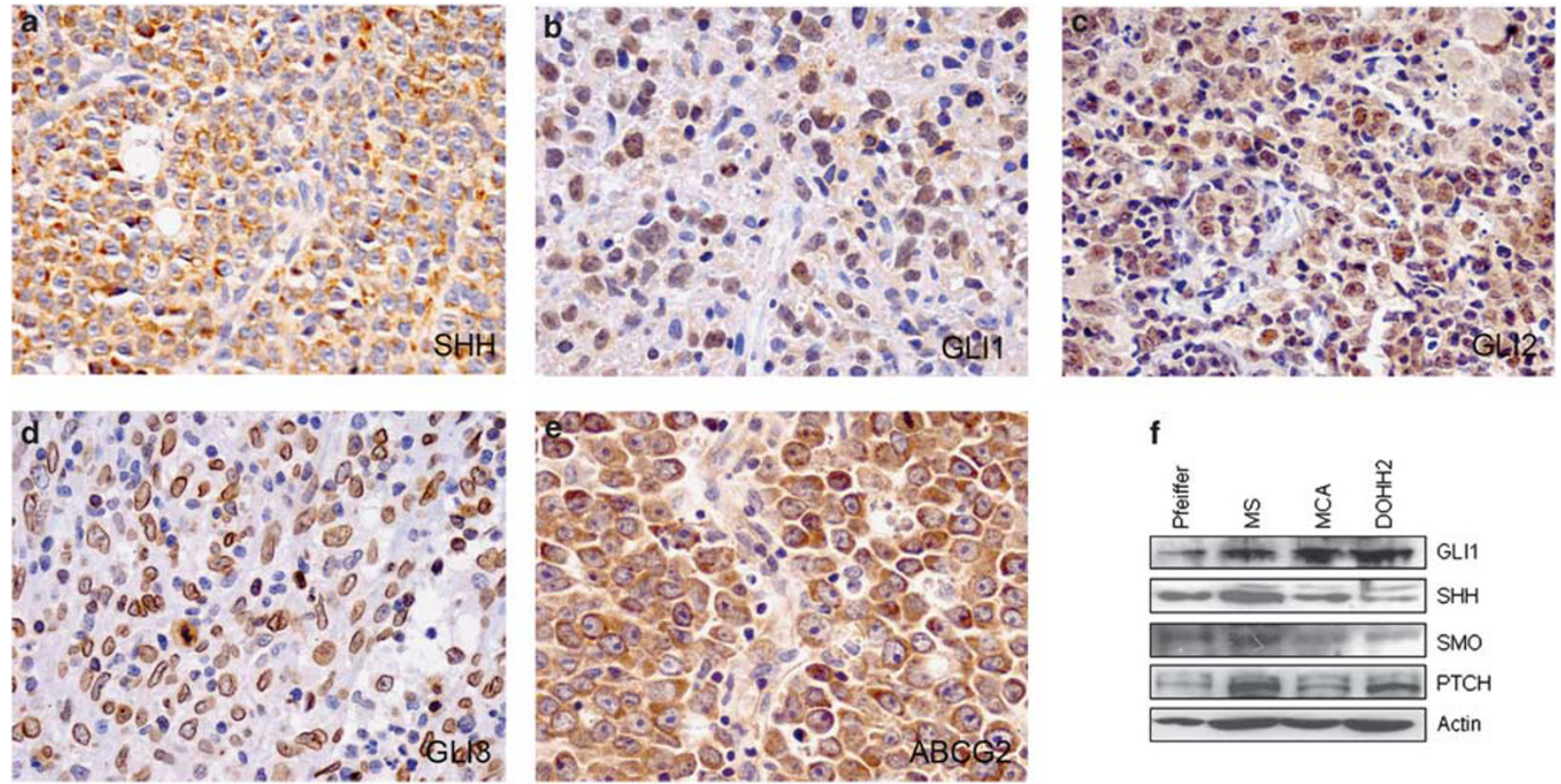

Figure 3 Sonic hedgehog signaling proteins and ABCG2 expression in diffuse large B-cell lymphoma. Cytoplasmic SHH expression is present in most diffuse large B-cell lymphomas, and high expression of SHH was shown in almost half of the cases in this study (a). Most diffuse large B-cell lymphomas express GLI1 (b) and GLI2 (c) in a cytoplasmic distribution. The tumor cells in a subset of diffuse large B-cell lymphoma cases expressed nuclear GLI3 (d). Cytoplasmic ABCG2 is expressed in most diffuse large B-cell lymphomas (e). (f) Western blots using whole-cell lysates of representative diffuse large B-cell lymphoma cell lines (DOHH2, MCA, MS, and Pfeiffer) show variable expression levels of GLI1, SHH, SMO, and PTCH.

Table 3 Correlation between SHH signaling proteins in diffuse large B-cell lymphoma

\begin{tabular}{lc}
\hline Markers & P-value \\
\hline GLI1-GLI2 & 0.001 \\
GLI1-GLI3 & 0.026 \\
GLI3-ABCG2 & 0.018 (negative) \\
SHH-ABCG2 & 0.05 \\
\hline
\end{tabular}

\section{Expression Levels of SHH Signaling Proteins and ABCG2 are Statistically Associated}

There was a significant positive correlation between the expression levels of GLI1 with GLI2 $(P=0.001)$ and with GLI3 $(P=0.026)$. There was a significant positive correlation between expression levels of SHH and ABCG2 $(P=0.05)$. There was a negative correlation between expression levels of GLI3 and ABCG2 $(P=0.018)$ (Table 3$)$.

\section{High expression levels of ABCG2 correlate with survival in DLBCL}

Patients with diffuse large B-cell lymphoma expressing high levels of ABCG2 showed a statistically significant shorter overall survival (5-year overall survival, $50 \%$; mean survival: 73 months) than patients with no or low levels of ABCG2 expression (5-year overall survival, $74 \%$; mean survival time:
159 months) ( $P=0.031)$ (Figure 4, left). Similarly, patients with diffuse large B-cell lymphoma expressing high levels of ABCG2 had inferior failure-free survival (5-year failure-free survival, $11 \%$; mean failure-free survival time: 21 months) than patients with tumors expressing low or no levels of ABCG2 (5-year failure-free survival, 39\%; mean failure-free survival time: 43 months $(P=0.028)$ (Figure 4, right; Table 4). No correlation between levels of expression of SHH signaling proteins and ABCG2 with other clinicopathological variables was noted, including immunophenotype (germinal center versus nongerminal center), high-grade features (as defined in Materials and methods), International Prognostic Index, and/or clinical stage.

\section{Discussion}

This is the first study showing aberrant high expression of SHH signaling proteins and ABCG2 in diffuse large B-cell lymphoma. The results also show that these proteins are not expressed or are expressed at only low levels in follicular lymphoma, chronic lymphocytic leukemia/small lymphocytic lymphoma, and non-neoplastic germinal center B cells.

The SHH signaling pathway is tightly regulated during adulthood and is restricted to specific organs, such as the skin, prostate gland, nervous system, and gastrointestinal tract, in which the SHH 

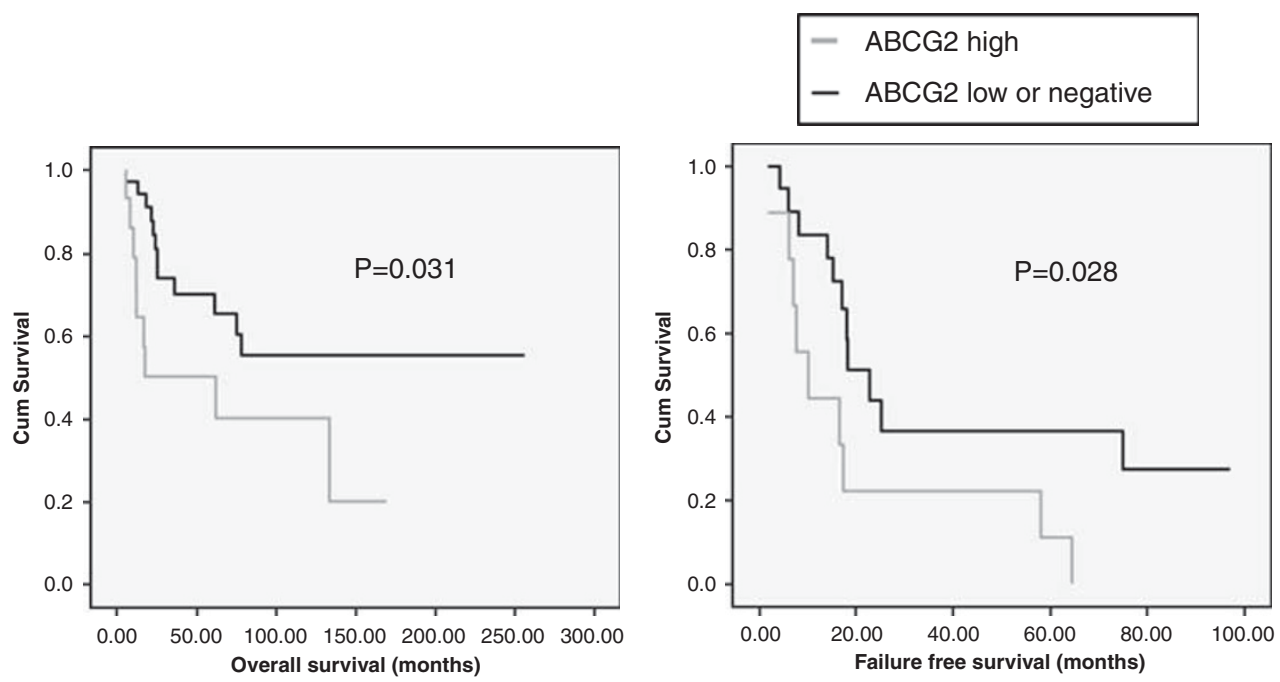

Figure 4 Overall survival (left) and failure-free survival (right) for patients with diffuse large B-cell lymphoma expressing high (green lines) levels of ABCG2 versus low or no ABCG2 expression (black lines). Patients with diffuse large B-cell lymphoma expressing high levels of ABCG2 showed significantly shorter overall survival $(P=0.031)$ and failure-free survival $(P=0.029)$ than patients with tumors with low or no expression of ABCG2.

Table 4 Comparison between expression of SHH signaling proteins with overall survival and failure-free survival in diffuse large B-cell lymphoma patients

\begin{tabular}{|c|c|c|c|c|c|c|}
\hline \multirow[t]{2}{*}{ Markers } & \multicolumn{2}{|c|}{ Mean overall survival (months) } & \multirow[t]{2}{*}{ P-value } & \multicolumn{2}{|c|}{ Mean failure-free survival (months) } & \multirow[t]{2}{*}{ P-value } \\
\hline & High & Low or no expression & & High & Low or no expression & \\
\hline SHH & 132 & 151 & 0.697 & 36 & 38 & 0.816 \\
\hline GLI1 & 93 & 161 & 0.261 & 35 & 40 & 0.659 \\
\hline GLI2 & 68 & 170 & 0.806 & 37 & 34 & 0.774 \\
\hline GLI3 $^{a}$ & 121 & 165 & 0.453 & 29 & 34 & 0.974 \\
\hline ABCG2 & 73 & 152 & $0.031^{\mathrm{b}}$ & 21 & 43 & $0.028^{\mathrm{b}}$ \\
\hline
\end{tabular}

${ }^{\mathrm{a}}$ For GLI3, cases were grouped as positive and negative.

${ }^{\mathrm{b}}$ Statistically significant.

pathway is involved in the maintenance of adult stem cells and production of the progeny that differentiates into specialized cell lineages. ${ }^{3,18-20}$ $\mathrm{SHH}$ also has been shown to regulate the proliferation and differentiation of hematopoietic precursor cells. In the thymus, $\mathrm{SHH}$ is a regulator of T-cell differentiation and T-cell receptor repertoire selection. ${ }^{4,21,22}$ In lymph nodes, Sacedon et $a l^{4}$ found that $\mathrm{SHH}$ is produced by follicular dendritic cells and that germinal center B cells express PTCH and SMO, components of the $\mathrm{SHH}$ receptor, suggesting that germinal center B cells are targets of SHH ligand produced by follicular dendritic cells. These authors also showed in vitro that $\mathrm{SHH}$ inhibition induces apoptosis in germinal center B cells, and that addition of exogenous SHH rescues germinal center B cells from Fas-induced apoptosis. ${ }^{4}$ These findings indicate that $\mathrm{SHH}$, in physiological conditions, is one of the survival signals provided by follicular dendritic cells to prevent apoptosis in germinal center B cells, and that SHH signaling plays mainly a paracrine role in the germinal center compartment.

Sonic hedgehog signaling plays a key role in tumorigenesis, and inappropriate activation of this pathway has been shown in many cancers including lymphomas and leukemias. Others ${ }^{23,24}$ have shown that SHH signaling has a paracrine role as a survival factor for B-cell lymphomas, including chronic lymphocytic leukemia/small lymphocytic lymphoma, and plasma cell neoplasms. These authors showed that hedgehog ligands secreted by bone marrow, nodal, and splenic stromal cells function as survival factors for malignant lymphomas and plasma cell myeloma. Recently, we have shown that SHH signaling is activated in ALK-positive anaplastic large cell lymphoma and that SHH activation mediates cell proliferation, cell survival, and contributes to the oncogenic effect of NPM-ALK in this lymphoma type. ${ }^{17}$

Here, we show that diffuse large B-cell lymphomas commonly express SHH, GLI1, and GLI2, at 
variable levels, and that a large percentage of these tumors express high levels of these proteins. This is not true for follicular lymphoma and chronic lymphocytic leukemia/small lymphocytic lymphoma. We also show that diffuse large B-cell lymphoma cell lines express SHH ligand, SHH receptors (PTCH and SMO), and the transcription factor GLI1. All these data indicate that diffuse large B-cell lymphoma tumor cells aberrantly express high levels of $\mathrm{SHH}$ signaling proteins and suggest that this aberrant expression may result in autocrine activation of the $\mathrm{SHH}$ pathway. It has been shown that $\mathrm{SHH}$, in a direct and/or indirect manner, upregulates the transcription of its own transcriptional effectors, including GLI1, suggesting the presence of positive autocrine loop with a positive feedback mechanism. ${ }^{7,8}$ These findings suggest that the SHH pathway may play an autocrine role in diffuse large B-cell lymphoma and provide a rationale for further studies investigating the pathogenetic role of SHH signaling in diffuse large B-cell lymphoma.

In this study, we also assessed diffuse large B-cell lymphoma for expression of ABCG2, one of the ABC proteins and a downstream target of $\mathrm{SHH}$ signaling. Multidrug resistance occurs when cancer cells acquire simultaneous resistance to a diverse class of chemotherapeutic agents with no structural or functional similarities. One mechanism by which tumor cells achieve multidrug resistance is by active efflux of chemotherapeutic agents through membrane drug transport proteins. ABC proteins act as ATP-dependent drug efflux pumps, which actively extrude certain classes of chemotherapeutic agents from cells. We report, for the first time, that ABCG2 is frequently expressed in diffuse large B-cell lymphomas and that the expression levels are generally higher than those detected in chronic lymphocytic leukemia/small lymphocytic lymphoma and follicular lymphoma. We also provide preliminary evidence that high expression of ABCG2 correlates with poorer prognosis and/or poor response to chemotherapy in patients with diffuse large B-cell lymphoma, and that ABCG2 proteins levels correlate with expression levels of SHH protein. Sims-Mourtada et $a l^{12}$ have shown that $\mathrm{SHH}$ signaling regulates the expression of $\mathrm{ABC}$ transporter proteins, including ABCG2, and that downregulation of ABCG2 expression by small interfering RNA partially reverses $\mathrm{SHH}$-induced chemoresistance. Other studies have shown that expression of $\mathrm{ABC}$ transporters in solid tumors predicts patients' poor response to chemotherapy. ${ }^{25,26}$ These findings suggest that ABCG2 may have an important biological role in diffuse large Bcell lymphoma, and that therapeutic inhibition of ABCG2 and/or the SHH signaling pathway may increase chemosensitivity and/or reverse chemoresistance in patients with diffuse large B-cell lymphoma.
In conclusion, our findings support the concept that the stroma is the main source of SHH protein in normal lymph nodes, and in lymph nodes involved by follicular lymphoma and chronic lymphocytic leukemia/small lymphocytic lymphoma. However, we found aberrant expression of $\mathrm{SHH}$ signaling proteins by diffuse large B-cell lymphoma cells, suggesting the possibility of an autocrine role of SHH signaling in the pathophysiology of diffuse large B-cell lymphoma. We have also shown that diffuse large B-cell lymphoma is characterized by high expression levels of ABCG2 in comparison with follicular lymphoma and with chronic lymphocytic leukemia/small lymphocytic lymphoma, and that the presence of high levels of ABCG2 in diffuse large B-cell lymphoma tumors correlates with shorter patient overall survival and failure-free survival.

\section{Conflict of interest}

The authors declare no conflict of interest.

\section{Acknowledgement}

This research was supported by funds from the University Cancer Foundation at The University of Texas MD Anderson Cancer Center.

\section{References}

1 Armitage JO, Weisenburger DD. New approach to classifying non-Hodgkin's lymphomas: clinical features of the major histologic subtypes. Non-Hodgkin's lymphoma classification project. J Clin Oncol 1998;16:2780-2795.

2 McMahon AP, Ingham PW, Tabin CJ. Developmental roles and clinical significance of hedgehog signaling. Curr Top Dev Biol 2003;53:1-114.

3 Bhardwaj G, Murdoch B, Wu D, et al. Sonic hedgehog induces the proliferation of primitive human hematopoietic cells via BMP regulation. Nat Immunol 2001;2:172-180.

4 Sacedon R, Diez B, Nunez V, et al. Sonic hedgehog is produced by follicular dendritic cells and protects germinal center B cells from apoptosis. J Immunol 2005;174:1456-1461.

5 Murone M, Rosenthal A, de Sauvage FJ. Hedgehog signal transduction: from flies to vertebrates. Exp Cell Res 1999;253:25-33.

6 Eichberger T, Sander V, Schnidar H, et al. Overlapping and distinct transcriptional regulator properties of the GLI1 and GLI2 oncogenes. Genomics 2006;87:616-632.

7 Dai P, Akimaru H, Tanaka Y, et al. Sonic hedgehoginduced activation of the Gli1 promoter is mediated by GLI3. J Biol Chem 1999;274:8143-8152.

8 Lee J, Platt KA, Censullo P, Ruiz i Altaba A. Gli1 is a target of Sonic hedgehog that induces ventral neural tube development. Development 1997;124:2537-2552.

9 Wang B, Fallon JF, Beachy PA. Hedgehog-regulated processing of Gli3 produces an anterior/posterior 
repressor gradient in the developing vertebrate limb. Cell 2000;100:423-434.

10 Gottesman MM, Fojo T, Bates SE. Multidrug resistance in cancer: role of ATP-dependent transporters. Nat Rev Cancer 2002;2:48-58.

11 Ambudkar SV, Dey S, Hrycyna CA, et al. Biochemical, cellular, and pharmacological aspects of the multidrug transporter. Annu Rev Pharmacol Toxicol 1999; 39:361-398.

12 Sims-Mourtada J, Izzo JG, Ajani J, Chao KS. Sonic hedgehog promotes multiple drug resistance by regulation of drug transport. Oncogene 2007;26:5674-5679.

13 Sims-Mourtada J, Izzo JG, Apisarnthanarax S, et al. Hedgehog: an attribute to tumor regrowth after chemoradiotherapy and a target to improve radiation response. Clin Cancer Res 2006;12:6565-6572.

14 Hans CP, Weisenburger DD, Greiner TC, et al. Confirmation of the molecular classification of diffuse large B-cell lymphoma by immunohistochemistry using a tissue microarray. Blood 2004;103:275-282.

15 Ford RJ, Goodacre A, Ramirez I, et al. Establishment and characterization of human B-cell lymphoma cell lines using B-cell growth factor. Blood 1990;75:1311-1318.

16 Vega F, Medeiros LJ, Leventaki V, et al. Activation of mammalian target of rapamycin signaling pathway contributes to tumor cell survival in anaplastic lymphoma kinase-positive anaplastic large cell lymphoma. Cancer Res 2006;66:6589-6597.

17 Singh RR, Cho-Vega JH, Davuluri Y, et al. Sonic hedgehog signaling pathway is activated in ALKpositive anaplastic large cell lymphoma. Cancer Res 2009;69:2550-2558.
18 Machold R, Hayashi S, Rutlin M, et al. Sonic hedgehog is required for progenitor cell maintenance in telencephalic stem cell niches. Neuron 2003;39:937-950.

19 Ramalho-Santos M, Melton DA, McMahon AP. Hedgehog signals regulate multiple aspects of gastrointestinal development. Development 2000;127:2763-2772.

20 Karhadkar SS, Bova GS, Abdallah N, et al. Hedgehog signalling in prostate regeneration, neoplasia and metastasis. Nature 2004;431:707-712.

21 Outram SV, Varas A, Pepicelli CV, Crompton T. Hedgehog signaling regulates differentiation from double-negative to double-positive thymocyte. Immunity 2000;13:187-197.

22 Rowbotham NJ, Hager-Theodorides AL, Cebecauer M, et al. Activation of the hedgehog signaling pathway in T-lineage cells inhibits TCR repertoire selection in the thymus and peripheral T-cell activation. Blood 2007;109:3757-3766.

23 Dierks C, Grbic J, Zirlik K, et al. Essential role of stromally induced hedgehog signaling in B-cell malignancies. Nat Med 2007;13:944-951.

24 Hegde GV, Peterson KJ, Emanuel K, et al. Hedgehoginduced survival of B-cell chronic lymphocytic leukemia cells in a stromal cell microenvironment: a potential new therapeutic target. Mol Cancer Res 2008;6:1928-1936.

25 Doyle LA, Ross DD. Multidrug resistance mediated by the breast cancer resistance protein BCRP (ABCG2). Oncogene 2003;22:7340-7358.

26 Chintamani, Singh JP, Mittal MK, et al. Role of pglycoprotein expression in predicting response to neoadjuvant chemotherapy in breast cancer-a prospective clinical study. World J Surg Oncol 2005;3:61. 